\title{
Das Ehrenamt macht Bibliotheken reicher
}

Zusammenfassung: Ausgehend von den langjährigen Erfahrungen der Städtischen Bibliotheken Dresden im Bereich Ehrenamt erläutern die Autoren die Bedingungen, unter denen Ehrenamt sowohl für die Einrichtung als auch für die Ehrenamtlichen selbst bereichernd ist. Nach einem kurzen historischen Abriss zum Ehrenamt in Dresden und Überlegungen zur Bedeutung von strategischen Partnern werden anschließend vier erfolgreiche Projekte vorgestellt, die nur durch die Unterstützung Ehrenamtlicher realisiert wurden und werden.

Schlüsselwörter: Ehrenamt, Öffentliche Bibliothek, strategische Partnerschaft, Leseförderung, aufsuchende Bibliotheksarbeit, Integration, Schulbibliothek

\section{Volunteering Makes Libraries Richer}

Abstract: Based on many years of experience of the Dresden Municipal Libraries in the field of volunteering, the authors explain the conditions under which volunteering is enriching the institution and the volunteers. After a short historical outline of volunteering in Dresden and the importance of strategic partners, four successful projects are presented. All projects can only be realized with the support of volunteers.

Keywords: Volunteering, public library, strategic partnership, reading literacy, outreach library work, integration, school library

\section{$1 \quad$ Einleitung}

2020: Im Corona-Jahr einen Artikel über das Ehrenamt in Bibliotheken zusammenzustellen, liegt nicht sofort auf der Hand.

Die Pandemie stellte und stellt alle gesellschaftlichen Bereiche vor nie erfahrene Herausforderungen, welche offene Auseinandersetzungen über die traditionellen und zukünftigen Aufgaben von Bibliotheken und die geeigneten Organisationsformen zu deren Bewältigung verlangen.

Die Einbindung des Ehrenamtes in den professionellen Bibliotheksbetrieb ist eine solche Diskussion wert, hochaktuell und nicht ohne Widersprüche:

- Wie passt die hauptamtliche Tätigkeit im Rahmen bewährter Berufsbilder zum ehrenamtlichen Engagement größtenteils berufsfremder Helferinnen und Helfer? 
Preprints 2021 der Zeitschrift BIBLIOTHEK - Forschung und Praxis, BFP.2020.0122 Flemming und

\section{Lippmann}

Dies ist ein Preprint $(\boldsymbol{c c})$ EY-NG-ND. Die endgültige Publikationsfassung erscheint beim Verlag De Gruyter unter https://www.degruyter.com/view/j/bfup

- Wie gelingt die organisatorische Einbindung von freiwilligen Tätigkeiten in unsere professionellen, zuverlässig anzubietenden Dienstleistungen?

- Was sind die Voraussetzungen erfolgreicher Ehrenamtsarbeit, wie koordiniert man diese Tätigkeiten, welche Qualifizierungsprogramme sind nötig?

- Welche Auswirkungen haben die Einschränkungen durch die Coronapandemie?

Dieser Artikel basiert auf den langjährigen Erfahrungen der Städtischen Bibliotheken Dresden, schaut auch in die sächsische Bibliotheksszene und verlässt den Bibliotheksbereich, um beengende Grenzen des öffentlichen Dienstrechts und der finanziellen Spielräume zu überwinden.

Öffentliche Bibliotheken (die wissenschaftlichen sind selbstverständlich öffentlich und hier ausdrücklich eingeschlossen) haben auch die schöne und dankbare Aufgabe, bürgerschaftliches Engagement anzuregen, aufzugreifen und professionelle Rahmenbedingungen zur Verwirklichung zu schaffen.

Ehrenamtliche Projekte haben in und für Bibliotheken mindestens drei strategische Vorteile:

- Die Bibliothek bietet ihre Räume, ihre Kompetenzen und ihr Aufgabenspektrum als Betätigungsfeld für Bürger*innen und ist damit Ort bürgerschaftlichen Engagements.

- Die Möglichkeiten der Angebote bibliothekarischer Serviceleistungen jenseits der tarifrechtlichen Tätigkeiten erweitern die Kernangebote der Bibliothek in für die Bürger*innen relevanten Bereiche.

- Ehrenamtlich Engagierte gehören zu den besten Stakeholdern der Bibliothek, ein hohes bürgerschaftliches Engagement ohne finanzielle Eigeninteressen ist ein überzeugendes Argument in der politischen Diskussion über die Aufgaben und notwendigen Ressourcen der Bibliothek.

\section{Ehrenamtsmanagements in den Städtischen Bibliotheken Dresden}

\subsection{Die Wurzeln}

Ehrenamtliche Traditionen, die auf bürgerlichen Entwicklungen im 19. Jahrhundert beruhen, gehen auch in Dresden bis in die bibliothekarischen Gründungsjahre zurück: Die Städtischen Bibliotheken wurden mit der Gründung der Dresdner Volksbibliotheken durch den „Gemeinnützigen Verein zur Förderung der sittlichen, geistigen und ökonomischen Interessen der Bevölkerung Dresdens“ ehrenamtlich betrieben.

Erst 1909 übernahm die Stadt die Bibliotheken vollständig in ihre Verwaltung, um die überwiegende öffentliche Finanzierung sicherzustellen.

Die für die aktuellen Entwicklungen relevanten Ansätze zum Neustart des ehrenamtlichen Engagements gehen auf die Zeiten der Eingemeindungen nach der politischen Wende 1990 zurück. In das etablierte und organisatorisch angemessen optimierte (reduzierte) Netz der Bibliotheken der Stadt waren die meist deutlich kleineren und in der Regel ehrenamtlich an wenigen Wochenstunden geöffneten Bibliotheken der eingemeindeten Ortschaften zu integrieren. Dies nahm die Bibliothek stadtweit zum Anlass, neu über die Einbindung von ehrenamtlich geleisteter Tätigkeit zu diskutieren. 
Preprints 2021 der Zeitschrift BIBLIOTHEK - Forschung und Praxis, BFP.2020.0122 Flemming und

\section{Lippmann}

Dies ist ein Preprint $(\boldsymbol{c c})$ EY-NG-ND . Die endgültige Publikationsfassung erscheint beim Verlag De Gruyter unter https://www.degruyter.com/view/j/bfup

Nach der in zahlreichen Diskussionen erreichten Überwindung von berufspolitischen Vorbehalten und der klaren Abgrenzung der unterschiedlichen Tätigkeitsbereiche erfolgte nicht nur die bis heute erfolgreiche Umsetzung des ehrenamtlichen Betriebs der eingemeindeten Bibliotheken, sondern es gelangen auch erste erfolgreiche Versuche der Einbindung ehrenamtlicher Helfer*innen in verschiedenen Stadtteilbibliotheken.

Darüber hinaus suchten die Städtischen Bibliotheken Dresden nach weiteren Möglichkeiten, um ein ansprechendes und für die Einrichtung zugleich unterstützendes Betätigungsfeld für an einem Ehrenamt interessierte Dresdner*innen zu initiieren. Unter dieser Maßgabe wurden in den vergangenen knapp zwölf Jahren vier umfangreiche Programme entwickelt, deren Aufgabenbereiche sehr unterschiedlich sind. Insgesamt engagieren sich hier 320 Dresdner und Dresdnerinnen ehrenamtlich. Alle Ehrenamtsmodule sind Ausdruck von sozialem Zusammenhalt und Teilhabegerechtigkeit.

\subsection{Bücherhausdienst}

Bereits 1996 wurde der Bücherhausdienst ins Leben gerufen, um Kranke, Hochbetagte oder Menschen mit Behinderungen zuhause mit Büchern und anderen Medien zu versorgen. Bis 2012 wurde der Bücherhausdienst durch befristet eingestellte ABM-Kräfte bzw. KommunalkombiMitarbeiter*innen gewährleistet. Bereits damals erwies sich die Partnerschaft mit der Bürgerstiftung Dresden als Voraussetzung für eine realisierbare Organisation.

Um die Weiterführung des bewährten Bücherhausdienstes nach Auslaufen der Maßnahmen des zweiten Arbeitsmarktes zu gewährleisten und sogar auszubauen, erfolgte von 2012 bis 2014 eine Neustrukturierung. Unterstützt wurde die Umwandlung des Bücherhausdienstes ins Ehrenamt vom Bundesministerium für Bildung und Forschung (BMBF) im Rahmen der Initiative „Lernen vor Ort“. Im September 2014 endete die Projektphase und der Ehrenamtliche Bücherhausdienst wurde in Dauerbetrieb überführt. Nun übernahmen ehrenamtliche Bücherbot*innen diese Aufgaben. Deren fachliche Anleitung und organisatorische Betreuung liegt in den Händen einer Mitarbeiterin der Städtischen Bibliotheken Dresden.

Die Einbindung von Ehrenamtlichen in die soziale Bibliotheksarbeit ging auch mit einer Dezentralisierung dieses Services einher. Starteten die Mitarbeiter*innen bis 2012 vom zentralen Depot der Sozialen Bibliotheksarbeit zu den Besuchen der immobilen Nutzer*innen, agieren die Ehrenamtlichen nunmehr aus ihrem Stadtteil heraus. Der Weg zwischen hilfsbereiten und hilfsbedürftigen Mitbürger*innen ist somit kurz. Die Bücherbot*innen wählen in Absprache mit den zu betreuenden Personen Medien aus und bringen diese zu innen nach Hause. Die Medienbereitstellung erfolgt in einer der 19 Stadtteilbibliotheken oder in der Zentralbibliothek. 
Preprints 2021 der Zeitschrift BIBLIOTHEK - Forschung und Praxis, BFP.2020.0122 Flemming und

\section{Lippmann}

Dies ist ein Preprint $(\boldsymbol{c c})$ EY-NG-ND . Die endgültige Publikationsfassung erscheint beim Verlag De Gruyter unter https://www.degruyter.com/view/j/bfup

Momentan betreuen 90 Bücherbot*innen ebenso viele Leser*innen. Ihre ehrenamtliche Arbeit ist von hoher sozialer und gesellschaftlicher Relevanz: Sie ermöglichen immobilen oder erkrankten Dresdner*innen den Zugang zu aktuellen Informations-, Bildungs- und Unterhaltungsmedien und somit eine höhere Lebensqualität.

\subsection{Lesestark! Dresden blättert die Welt auf}

Bildungsunterstützung, insbesondere Leseförderung für Kinder, gehört seit Jahren zu den Kernaufgaben der Städtischen Bibliotheken Dresden. In den vergangenen Jahren wurde das Angebot in diesem Bereich stark erweitert: Ausbau des Bestandes an Kinderliteratur, Verstärkung der Veranstaltungstätigkeit im kinderliterarischen Bereich, Verschiebung von Personalkapazitäten, um den gestiegenen Arbeitsaufwand zu bewerkstelligen.

Im Herbst 2008 starteten die Städtischen Bibliotheken Dresden dann mit „Lesestark! Dresden blättert die Welt auf.", ein großangelegtes Projekt zur Lese- und Sprachförderung von Vor- und Grundschulkindern, welches in seiner angedachten Dimension die Zusammenarbeit mit starken Partnern unverzichtbar machte. Mit der Drosos Stiftung Zürich, die sich weltweit sozial engagiert, und der Bürgerstiftung Dresden, wichtigste Förderin des Ehrenamts in der Region, wurden diese Mitstreiter gefunden.

„Lesestark!“ richtet sich an Kinder im Übergangsalter vom Vorschulkind zum Erstleser sowie an deren Eltern und Betreuungspersonen. Ziel des Leseförderprogrammes ist es, durch eine breit angelegte Leseförderung sowie eine hohe Betreuungsdichte bei Kindern die Leselust zu wecken und auf diesem Weg die Kernkompetenzen Sprach- und Lesefähigkeiten bei den am Projekt teilnehmenden Kindern verbessern zu helfen, um deren Bildungschancen nachhaltig zu erhöhen.

Um dieses Ziel zu erreichen, kommt den ehrenamtlichen Vorlesepat*innen eine zentrale Rolle zu. Jede/r ehrenamtliche Lesepat*in begleitet für mindestens ein Schuljahr eine oder mehrere Vorschulgruppen bzw. Schulklassen. Dort wird wenigstens einmal im Monat vorgelesen. Um die Anbindung der Gruppen/Klassen an die jeweilige Statteilbibliothek zu gewährleisten, finden die Vorlesestunden sowohl im Kindergarten bzw. in der Schule als auch in der Bibliothek statt.

Flankiert wird das Vorlesen von Honorarveranstaltungen externer Partner, die Sachthemen für die Zielgruppen in enger Verknüpfung mit Kinderliteratur präsentieren. Das Themenspektrum reicht dabei von Stadtrundgängen durch Dresden über Bionik bis hin zum Umweltschutz.

1.508 Vorschulkinder und 687 Grundschulkinder nahmen 2008 am Programm „Lesestark" teil. Im Schuljahr 2019/2020 waren es 1.984 Kinder im Vorschulbereich und 3.162 im Grundschulbereich. Solche Zuwächse waren nur möglich, weil sich die Zahl der Lesepat*innen im gleichen Zeitraum von 
Preprints 2021 der Zeitschrift BIBLIOTHEK - Forschung und Praxis, BFP.2020.0122 Flemming und

\section{Lippmann}

Dies ist ein Preprint $(\boldsymbol{c c})$ EY-No-ND. Die endgültige Publikationsfassung erscheint beim Verlag De Gruyter unter https://www.degruyter.com/view/j/bfup

57 auf 140 erhöhte. Diese anspruchsvolle ehrenamtliche Arbeit erfreut sich offenbar großer Beliebtheit. Den Kindern Lesefreude zu vermitteln, liegt vielen Dresdner*innen am Herzen. Sie bringen ihre ganz individuellen Erfahrungen mit Kindern und mit Kinderliteratur ein.

Die Akquise der Ehrenamtlichen erfolgt durch das hauptamtliche Team „Lesestark“. Die Mitarbeiter*innen laden Interessierte zu einem leitfadengestützten Auswahlgespräch ein, in welchem Fertigkeiten, Interessen und Motive der Bewerber*innen ausgelotet werden. Bereits bei diesem ersten persönlichen Kontakt startet die umfangreiche und intensive Betreuung durch das Projektteam. Sind die Bewerber*innen für die ehrenamtliche Tätigkeit geeignet, unterzeichnen sie eine Kooperationsvereinbarung mit dem Programm Lesestark!. Voraussetzung für eine ehrenamtliche Tätigkeit ist die Vorlage des erweiterten polizeilichen Führungszeugnisses.

Der Einsatz der Ehrenamtlichen erfolgt möglichst wohnortnah. Die Mitarbeiter*innen der jeweiligen Stadtteilbibliothek verstehen sich als Ansprechpartner für die Literaturauswahl und organisatorische Fragen.

Unter dem Motto „Initiieren - Qualifizieren - Motivieren“ sind die Ehrenamtlichen in ein speziell auf ihre Bedürfnisse zugeschnittenes Fortbildungsprogramm eingebunden. Es ermöglicht ihnen, ihre Tätigkeit in Kindergärten, Grundschulen und Bibliotheken kompetent auszuführen. Ein jährlich wechselnder Themenschwerpunkt setzt immer wieder neue Akzente bzgl. der Angebote. Zugleich gibt es wiederkehrende Module wie Sprech- und Stimmtraining, Verlagspräsentationen und Vorstellen neuer Kinderliteratur. Diese sind als Basisschulung gedacht.

Das kostenlose Fortbildungsprogramm ist ein Teil der Anerkennungskultur der Städtischen Bibliotheken Dresden. Die hochwertigen Angebote (u.a. gemeinsame Fahrt zur Buchmesse, Besuch aktueller Ausstellungen in Dresdner Museen, gemeinsamer Besuch von Theatervorstellungen) werden von den Lesepat*innen als Wertschätzung ihres Engagements verstanden.

Darüber hinaus werden alle Ehrenamtlichen zu einem großen Sommerfest (s. Abb. 1) und zu einer gemeinsamen Weihnachtsfeier eingeladen. Ein künstlerischer Teil (z.B. Theater, Pantomime, Musik) bildet immer den Auftakt. Ein gemeinsames Abendbrot bietet ausreichend Zeit, um miteinander ins Gespräch zu kommen. 
Preprints 2021 der Zeitschrift BIBLIOTHEK - Forschung und Praxis, BFP.2020.0122 Flemming und

\section{Lippmann}

Dies ist ein Preprint $(\mathrm{cc})$ EY-NG-ND. Die endgültige Publikationsfassung erscheint beim Verlag De Gruyter unter https://www.degruyter.com/view/j/bfup

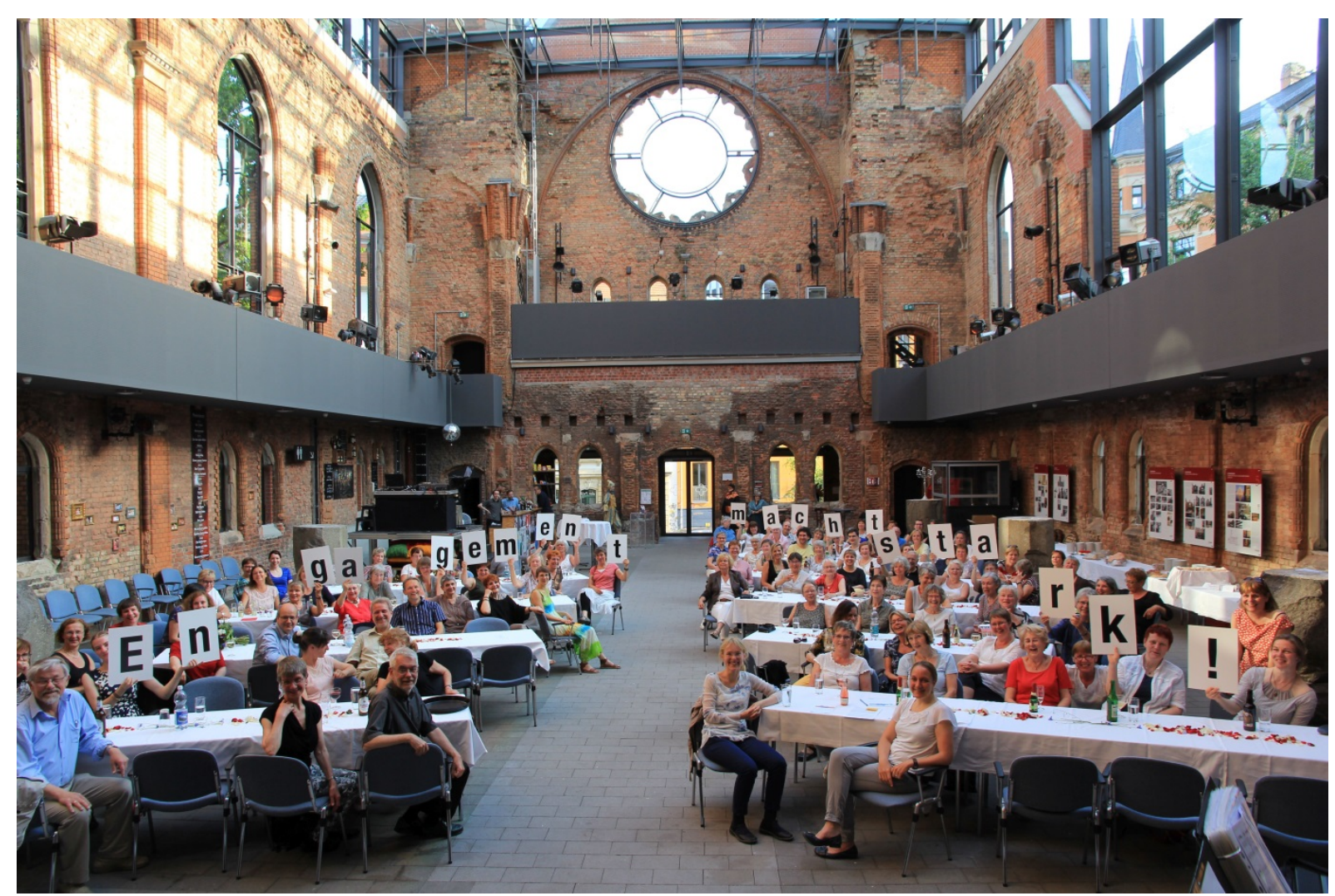

Abb. 1: Sommerfest der Ehrenamtlichen mit Aktion „Engagement macht stark“

In Kleingruppen finden zudem auch in den Stadtteilbibliotheken „Lesepatenfrühstücke“ statt. Dort können die Teilnehmer*innen in kleiner Runde in Austausch treten und auch eventuelle Probleme besprechen. Aber auch das Koordinations-Team versteht sich als Ansprechpartner für Fragen und bei Konflikten.

Ein weiterer Baustein einer Kultur der Anerkennung ist die Bereitstellung von Fahrscheinen der Dresdner Verkehrsbetriebe. In Abhängigkeit von der Anzahl der zu betreuenden Gruppen/Klassen erhalten die Lesepat*innen zweimal jährlich eine ausreichende Anzahl, um zu ihren Einsatzorten zu gelangen.

Das Leseförderprogramm „Lesestark“ ist das älteste und umfangreichste Ehrenamtsmodul der Städtischen Bibliotheken Dresden und verfügt somit über große Erfahrungen im Bereich Ehrenamtsmanagement. Diese Erfahrungen lassen sich in zehn Qualitätskriterien (s. $A b b .2)$ für die Betreuung ehrenamtlicher Lesepaten zusammenfassen. 
Preprints 2021 der Zeitschrift BIBLIOTHEK - Forschung und Praxis, BFP.2020.0122 Flemming und

\section{Lippmann}

Dies ist ein Preprint $(\boldsymbol{C c})$ EY-NG-ND. Die endgültige Publikationsfassung erscheint beim Verlag De Gruyter unter https://www.degruyter.com/view/j/bfup

1. Leitfadengestütztes Auswahlgespräch

2. Kooperationsvereinbarung und umfassende Informationsmaterialien

3. Impulsschulung Stimm- und Vorlesetechnik

4. Monatliche Fortbildungsangebote mit jährlich wechselndem Schwerpunkt (u.a. Literaturempfehlungen und Vermittlungsformen, Verlagspräsentationen)

5. Transparente und frühzeitige Terminplanung mit allen Partnern (Lesepat*innen, Kita/Grundschule, Bibliothek, Koordinationsteam)

6. Frühzeitige inhaltliche wie organisatorische Abstimmung der Themen

7. Transparenz und Abstimmung gegenseitiger Erwartungen bezüglich inhaltlicher Freiräume und Gestaltungsmöglichkeiten

8. Kultur der Anerkennung (Lesepatenfrühstück, Feste, Fahrscheine für ÖPNV)

9. Zeitnahes Beschwerdemanagement durch Koordinationsteam

10. Alternierendes strukturiertes Feedbackverfahren

Abb. 2: 10 Qualitätskriterien für das Ehrenamt im Projekt „Lesestark!“

\subsection{Dialog in Deutsch:}

Gelingende Integration von Zugewanderten wird maßgeblich von deren Kenntnis der Deutschen Sprache geprägt. Bibliotheken bieten mit ihren vielfältigen Medienbeständen die Möglichkeit, den Spracherwerb zu unterstützen. Als öffentliche, politisch und religiös neutrale, nichtkommerzielle und zudem geschützte Orte sind sie besonders geeignete Anlaufstellen für Zugewanderte.

Spätestens mit der verstärkten Migration seit Herbst 2015 entwickelten Kultur- und Bildungseinrichtungen neue Formate, um die Integration von Zugewanderten zu befördern. Die Städtischen Bibliotheken Dresden orientierten sich hierbei an den langjährigen Erfahrungen der Bücherhallen Hamburg und adaptierten das Konzept „Dialog in Deutsch“.

Seit April 2017 finden wöchentlich Gesprächsrunden statt, bei denen Zugewanderte die Möglichkeit haben, ihre Deutschkenntnisse im lockeren Gespräch anzuwenden und zu trainieren. In offener Atmosphäre können Erfahrungen ausgetauscht und neue Kontakte geknüpft werden. Die Freude am Deutsch-Sprechen steht dabei im Vordergrund. Die Gesprächsgruppen dauern jeweils eine Stunde und werden von zwei geschulten ehrenamtlichen Moderator*innen geleitet.

Diese sind - wie alle anderen ehrenamtlich Tätigen - in ein speziell auf ihre Bedürfnisse zugeschnittenes Fortbildungsprogramm eingebunden. Es umfasst Schulungen zu den Themen Interkulturelle Kompetenz, Meinungsaustausch sowie zur Gesetzeslage im Bereich Asylrecht. 
Preprints 2021 der Zeitschrift BIBLIOTHEK - Forschung und Praxis, BFP.2020.0122 Flemming und

\section{Lippmann}

Dies ist ein Preprint (cc) EY-No-ND. Die endgültige Publikationsfassung erscheint beim Verlag De Gruyter unter https://www.degruyter.com/view/j/bfup

In der Zentralbibliothek und in drei Stadtteilbibliotheken werden in 2020 vier Gesprächsgruppen von 40 Moderator*innen betreut. Interessant ist die Altersstruktur dieser Ehrenamtlichen. Während sich in den anderen Ehrenamtsprogrammen der Bibliothek häufig Frauen und Männer engagieren, die bereits aus dem Berufsleben ausgeschieden sind, engagieren sich hier viele Berufstätige und junge Dresdner*innen. Ein Grund dafür dürfte sein, dass das Format „Dialog in Deutsch“ in den Nachmittagsstunden bzw. an Sonnabendvormittagen stattfindet. Dieses Ehrenamt lässt sich daher besser in die Tagestruktur der Studierenden und Arbeitenden integrieren.

\subsection{Schulbibliotheken $\mathbf{2 0 2 5}$}

Die Städtischen Bibliotheken Dresden konnten in den letzten zwölf Jahren die Entleihungen an Kinderbüchern um mehr als 50 Prozent steigern und überschritten 2018 erstmalig die Millionenmarke. In keiner anderen Bestandsgruppe der physischen Medien ist eine solche Steigerungsrate zu verzeichnen. Diese Zuwächse sind das Ergebnis einer jahrelangen, klar strukturierten Kinderbibliotheksarbeit und des Leseförderprogramms „Lesestark! Dresden blättert die Welt auf".

Jährlich finden in den 19 Zweigstellen und der Zentralbibliothek ca. 5.000 Veranstaltungen für Kinder und Jugendliche statt. Ein Großteil davon ist für Schulklassen vorgesehen, die diese Anregungen gern und häufig nutzen und die jeweilige Stadtteilbibliothek bzw. die Zentralbibliothek aufsuchen. Die Bibliotheken sind somit ein wichtiger außerschulischer Lernort und haben insbesondere mit ihren literarischen und literaturpädagogischen Angeboten (Lesungen mit Autor*innen; Lesefeste, mobile Theateraufführungen, Vorlesestunden etc.) in der Dresdner Kultur- und Bildungslandschaft ein Alleinstellungsmerkmal.

Diese erfolgreiche Kinderbibliotheksarbeit mündete in der Idee, die Lehrer*innen in ihrer pädagogischen Arbeit durch die Einrichtung von Schulbibliotheken zu unterstützen. Die in den Vorjahren gemachten positiven Erfahrungen mit ehrenamtlicher Arbeit führten_seitens der Städtischen Bibliotheken zu der Überlegung, beim Aufbau von Grundschulbibliotheken einen besonderen Weg zu gehen. Während der Bestand, der sich am sächsischen Grundschullehrplan für Klasse drei und vier orientiert, von den Fachlektor*innen der Städtischen Bibliotheken Dresden zusammengestellt wird, sichern Ehrenamtliche die Öffnungszeiten ab. Für jede Grundschulbibliothek wird ein aus zwei bis drei Personen bestehendes Team gebildet. Alle sogenannten Leselotsen*innen werden von einer festangestellten Projektkoordinatorin unterstützt.

Die Betreibung einer Grundschulbibliothek in Dresden würde somit ein Zusammenspiel aus professioneller Bibliotheksarbeit und ehrenamtlichem Engagement sein. Ein solches Modell stieß im Dresdner Stadtrat auf großes Interesse, da eine wichtige bildungspolitische Aufgabe zu 
Preprints 2021 der Zeitschrift BIBLIOTHEK - Forschung und Praxis, BFP.2020.0122 Flemming und

\section{Lippmann}

Dies ist ein Preprint $(\boldsymbol{c c})$ EY-NG-ND. Die endgültige Publikationsfassung erscheint beim Verlag De Gruyter unter https://www.degruyter.com/view/j/bfup

vergleichsweise niedrigen Kosten (5.000 Euro pro Jahr und Schulbibliothek sowie eine Personalstelle für je 20 Schulbibliotheken) erfüllt werden konnte.

Mit einem überfraktionellen Stadtratsbeschluss erhielten die Städtischen Bibliotheken Dresden den Auftrag und auch die entsprechenden personellen und finanziellen Ressourcen, um pro Jahr zehn Schulbibliotheken zu eröffnen. Mittlerweile haben in 25 Grundschulen 50 ehrenamtliche Leselotsen*innen ihre Arbeit aufgenommen. Sie fungieren sowohl als Ansprechpartner*innen für die Kinder, welche die Bibliothek aufsuchen, als auch als Bindeglied zwischen der Schule bzw. dem Hort und den Städtischen Bibliotheken Dresden.

Inwieweit Ehrenamtliche in weiterführenden Schulen (Gymnasien, Oberschulen, Förderschulen) als Leselotsen*innen zum Einsatz kommen werden, ist derzeit noch nicht absehbar. Die Herausforderungen an diese Personen werden besonders sein: größere Fachlehrerschaft, Spezialisierungen der Schulen (Profile), Akzeptanz durch die jugendliche Schülerschaft usw.

Die Ergebnisse der ersten Pilotschulen sollen 2021 ausgewertet werden.

\section{Die Bedeutung von strategischen Partnern des Ehrenamts}

Das Strukturmodell Ehrenamtsmanagement der Städtischen Bibliotheken Dresden ist von zahlreichen Partnerschaften geprägt. Diese sind unverzichtbar für

- Einbindung in der Stadtgesellschaft

- Knüpfung von Kultur- und Bildungsnetzwerken

- Bündelung von inhaltlichen und organisatorischen Kompetenzen

- Koordination der relevanten Bedarfe und dafür angebotenen Dienstleitungen

- Akquise von am Ehrenamt interessierten Personen

- Abstimmung mit vergleichbaren Anbietern bürgerschaftlichen Engagements

- Fundraising zur Absicherung notwendiger Budgets

- breit angelegte Qualifikations- und Würdigungskonzepte.

Aus den Erfahrungen erfolgreicher Projekte entwickelte sich in den Städtischen Bibliotheken Dresden ein auf drei Säulen stehendes Strukturmodell:

\subsection{Projektträger Bibliothek}

- Die Städtischen Bibliotheken sind prinzipiell der Träger des Projektes und damit für die inhaltliche Ausrichtung, die rechtliche Einordnung und alle weiteren Aspekte voll verantwortlich.

- Die Finanzierung der professionellen Basis und aller fachlicher Aufwendungen erfolgt über das Budget der Bibliothek.

- Jedes Ehrenamtsprojekt verfügt über eine professionelle Basis im Rahmen des für die jeweiligen Inhalte und Services dienstlich zuständigen Personals der Bibliothek. Hier liegt auch die persönliche Verantwortung in den Händen der Führungskräfte der Bibliothek.

- Zur Betreuung der ehrenamtlichen Mitarbeiter*innen ergibt die Erfahrung, dass für jeweils 100 Freiwillige eine Stelle bzw. ein/e Mitarbeiter*in der Bibliothek zur Verfügung stehen sollte. 
- Das Qualifizierungs- und Würdigungskonzept ist genauso Aufgabe der Bibliothek wie die lückenlose Absicherung der Ansprechbarkeit für Anfragen und Probleme.

\subsection{Projektpartner Ehrenamt Bürgerstiftung Dresden}

- Die Bürgerstiftung ist projektübergreifend die Partnerin des Ehrenamtsmanagements der Städtischen Bibliotheken Dresden.

- Die Stabilisierung und Qualifizierung der Verbindung mit der Bürgerstiftung erfolgt durch das ehrenamtliche Engagement der Bibliotheksleitung im Stiftungsrat.

- Der im März 2000 von der Bürgerstiftung gegründete „Treffpunkt Hilfsbereitschaft“ wurde mit Unterstützung der Bibliothek 2019 zur Freiwilligenagentur „ehrensach.jetzt" profiliert. Diese ermöglicht stadtweit sowohl in persönlicher Beratung als auch mittels eines Onlineportals die Vermittlung von bürgerschaftlichem Engagement in allen Bereichen. Davon profitieren selbstverständlich auch die bibliothekarischen Projekte, da keine eigenen Strukturen aufzubauen sind und interessierte Bürger*innen, die sich nach den Erstkontakten nicht für eines der Projekte der Bibliothek entscheiden, auf andere Einsatzfelder aufmerksam gemacht werden können.

- Durch die gemeinsame Trägerschaft von Ehrenamtsprojekten gelingt es in Partnerschaft mit einer gemeinnützigen Stiftung gut, im Fundraising (gerade in Bezug auf finanzielle Partner, die nur nichtöffentliche Träger unterstützen dürfen) und in der Finanzorganisation (Flexibilität des Einsatzes der Mittel im Würdigungskonzept, Übertragbarkeit u.a.) die Grenzen der öffentlichen Verwaltung zu überschreiten. Als Partner der Bibliothek werden dieser die entstehenden Aufwände z. B. für die Finanzverwaltung nicht in Rechnung gestellt.

- Die Bürgerstiftung organisiert das sachsenweite Projekt „Wir für Sachsen“, das pauschale Aufwandsentschädigungen im Ehrenamt ermöglicht/unterstützt, daher wäre die Beteiligung der in den Bibliotheken tätigen Freiwilligen unbürokratisch zu ermöglichen. Allerdings ist das Interesse unserer Ehrenamtlichen an der Zahlung einer Aufwandsvergütung eher die Ausnahme und daher äußerst gering.

\subsection{Projektträger der einzelnen Projekte}

- Für jedes Projekt sucht die Bibliothek (in der Regel eine/n) geeignete/n Projektpartner*in, der/die sich mit den Zielen und Inhalten des Projektes identifiziert und eigene Ressourcen und Kompetenzen einbringt.

- Basis ist eine projektvertragliche Vereinbarung mit der Bibliothek, die in der Regel auch den Projektpartner Bürgerstiftung benennt, z. B. zur gemeinnützigen Verwaltung des Budgets.

- Ziel dieser einzelnen Partner ist, sowohl die Breite der öffentlichen Aufmerksamkeit zu erhöhen als auch das notwendige Budget für das Würdigungskonzept für die ehrenamtlich Tätigen bereitzustellen. Als finanzielle Basis für die Ermöglichung der ehrenamtlichen Projektanteile haben sich 100 Euro pro Jahr und je freiwillig tätiger Person bewährt.

\section{Ehrenamt in Sächsischen Bibliotheken im Coronajahr 2020}

Der Landesverband Sachsen im Deutschen Bibliotheksverband ermuntert seine Mitglieder seit vielen Jahren, mutig ins Ehrenamt zu investieren. So ermöglichte das Projekt „Lesestarkes Sachsen“ einen kommunikativen Austausch von Leseförderinitiativen im ganzen Freistaat Sachsen.

Die Coronaeinschränkungen des Bibliotheksbetriebs seit März 2020, viel mehr jedoch die noch immer notwendigen Kontaktbeschränkungen in allen Lebensbereichen, haben zu einer deutlichen Reduzierung der Möglichkeiten von ehrenamtlichen Tätigkeiten geführt. 
Preprints 2021 der Zeitschrift BIBLIOTHEK - Forschung und Praxis, BFP.2020.0122 Flemming und

\section{Lippmann}

Dies ist ein Preprint $(\boldsymbol{c c})$ EY-No-ND. Die endgültige Publikationsfassung erscheint beim Verlag De Gruyter unter https://www.degruyter.com/view/j/bfup

Eine Umfrage unter sächsischen Bibliotheken zur Vorbereitung dieses Artikels (August 2020, 77

Bibliotheken beteiligten sich an der Beantwortung) ergab folgende Situation:

- $60 \%$ der Bibliotheken verfügen über ehrenamtliche Unterstützung

- $48 \%$ der Bibliotheken ohne Ehrenamt gaben an, im regionalem Umfeld nicht genügend persönliche Angebote zu haben (26\% keinen Bedarf, 19 \% fehlende Genehmigung durch den Bibliotheksträger!)

- die Haupttätigkeitsbereiche sind:

$>$ Die Leseförderung (65\%)

$>$ Betreuung von Veranstaltungen (62\%)

$>$ Aufsicht während der Öffnungszeiten (24\%)

$>$ aufsuchende Bibliotheksarbeit (24\%)

$>$ Betreuung von Schulbibliotheken (18\%)

$>$ Engagement in Fördervereinen (15\%)

$>$ Integrationsarbeit (12\%).

Die Coronaeinschränkungen haben das Ehrenamt in sächsischen Bibliotheken aktuell um 20 Prozent reduziert. Viele Ehrenamtliche signalisierten, dass sie ihre Tätigkeit gern so schnell wie möglich fortsetzen möchten.

Eine wichtige Aufgabe der Projektkoordinator*innen im Bereich Ehrenamt wird es sein, unter den gegenwärtigen Bedingungen nach Lösungen zu suchen, die es Ehrenamtlichen erlauben, ihre Arbeit wiederaufzunehmen. Dabei müssen geltende Hygienevorschriften eingehalten werden. Der Gesundheitsschutz der Ehrenamtlichen steht dabei im Vordergrund. Die Ausstattung mit entsprechenden Hilfsmitteln (Mund-Nasen-Schutz, Gesichtsvisiere, Desinfektionsmittel usw.) ist genauso wichtig wie die persönliche Absprache mit Ehrenamtlichen, das Ehrenamt evtl. für einen befristeten Zeitraum auszusetzen, insbesondere wenn sie zu Risikogruppen gehören.

Über 90 Prozent aller Bibliotheken rechnet mit einer Rückkehr der Engagierten nach Beendigung der Pandemievorsorge, einige planen einen Ausbau der Projekte mit bürgerschaftlichem Engagement.

\section{Fazit: Erfolgsvoraussetzungen für gelingendes Ehrenamt}

- Die Einsatzgebiete müssen sich für freiwillige Tätigkeit eignen, sollten aber unbedingt im Kernbereich der Bibliothek angesiedelt sein. Freiwillig Engagierte müssen eine befriedigende, sinnvolle Tätigkeit finden, die als hochwertiger Mosaikstein der Bibliotheksdienstleistungen wahrgenommen werden kann.

- Das Ehrenamt muss professionell gemanagt, d.h. in den Rahmen der hauptamtlichen Struktur der Bibliothek eingebettet sein.

- Ehrenamtlich Tätige müssen themen- und bedarfsübergreifend ständige Ansprechpartner*innen in flachen, übersichtlichen Hierarchien haben.

- Arbeitsrechtliche Rahmenbedingungen sind unbedingt angemessen zu berücksichtigen:

$>$ Grundlage ist ein gemeinnütziges, unentgeltliches, zusätzliches, freiwilliges Engagement.

$>$ Ehrenamtliche Tätigkeiten gelten als nachrangig zu anderen entgeltlichen Beschäftigungsmöglichkeiten. 


\section{Lippmann}

Dies ist ein Preprint $(\boldsymbol{c})$ ) EY-No-ND . Die endgültige Publikationsfassung erscheint beim Verlag De Gruyter unter https://www.degruyter.com/view/j/bfup

$>$ Es existiert keine Weisungsbefugnis.

$>$ Verbindliche Vorgaben, zum Beispiel zur Einsatzzeit, sind kaum möglich.

$>$ Die Einsatzzeit ist in der Regel begrenzt.

Freiwilliges Engagement kann jederzeit ohne Angaben von Gründen beendet werden.

$>$ Versicherungsrechtliche Rahmenbedingungen sind zwingend zu beachten.

- Um arbeitsrechtliche Schwierigkeiten zu vermeiden und die Akzeptanz bei Personalämtern zu erhöhen, empfiehlt sich der Abschluss einer Vereinbarung zur Freiwilligentätigkeit mit dem Hinweis, dass sich kein Arbeits- oder Ausbildungsverhältnis daraus ableitet.

- Bei der Ermöglichung und Organisation des Ehrenamts müssen die unterschiedlichen Motive für freiwillige, unentgeltliche Tätigkeit Berücksichtigung finden:

$>$ Übernahme sozialer Verantwortung in altruistischer Motivation

$>$ Suche nach Sozialkontakten

$>$ Suche nach Lernmöglichkeiten und neuen Erkenntnissen und Fähigkeiten, sowohl zur Karriereförderung als auch nach Abschluss der beruflichen Tätigkeiten

$>$ Suche nach Selbsterfahrung und Bewältigung persönlicher Probleme.

Dies muss, neben der persönlichen Eignung für die konkrete freiwillige Tätigkeit, eine wesentliche Basis von Auswahl, Förderung und Begleitung (und ggf. auch Ablehnung) der Ehrenamtlichen sein.

- Die Qualifikation von Ehrenamtlichen ist für die Qualität der geleisteten Tätigkeit von großer Bedeutung, wird darüber hinaus von den Freiwilligen meist als bedeutende Investition in ihre Persönlichkeit verstanden und ist damit unverzichtbarer Bestandteil des Würdigungskonzeptes.

- Das Würdigungskonzept muss Teil eines offenen Kommunikationsplans sein.

Gesprächsorientierte Begegnungs- und Austauschformate mit anderen freiwillig Engagierten der Bibliothek sind genauso wichtig wie gemeinsame kulturelle Erlebnisse (in Dresden z. B. die Teilnahme am Neujahrsempfang des Oberbürgermeisters in der Philharmonie) über die Bibliothek hinaus.

- Die finanziellen und organisatorischen Grundlagen für eine angemessene Planungssicherheit sind unverzichtbar für eine erfolgreiche Arbeit.

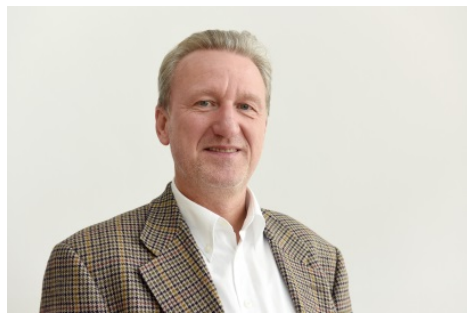

Prof. Dr. Arend Flemming

Städtische Bibliotheken Dresden

Schloßstr. 2

D-01067 Dresden

flg@bibo-dresden.de 
Preprints 2021 der Zeitschrift BIBLIOTHEK - Forschung und Praxis, BFP.2020.0122 Flemming und

\section{Lippmann}

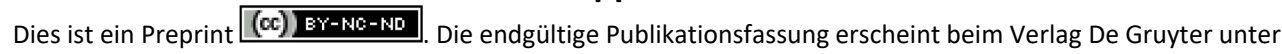
https://www.degruyter.com/view/j/bfup

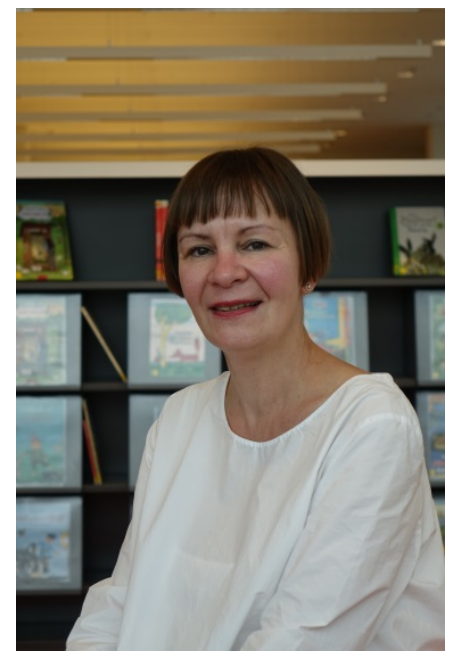

\section{Christine Lippmann}

Städtische Bibliotheken Dresden

SG Kulturelle Bildung und Integration

Schloßstr. 2

D-01067 Dresden

c.lippmann@bibo-dresden.de 